\title{
Mouse WRN Helicase Domain Is Not Required for Spontaneous Homologous Recombination-Mediated DNA Deletion
}

\author{
Adam D. Brown, ${ }^{1,2}$ Alison B. Claybon, ${ }^{1}$ and Alexander J. R. Bishop ${ }^{1,2}$ \\ ${ }^{1}$ Greehey Children's Cancer Research Institute, The University of Texas Health Science Center at San Antonio, \\ San Antonio, TX 78229, USA \\ ${ }^{2}$ Department of Cellular and Structural Biology, The University of Texas Health Science Center at San Antonio, \\ San Antonio, TX 78229, USA \\ Correspondence should be addressed to Alexander J. R. Bishop, bishopa@uthscsa.edu \\ Received 16 May 2010; Accepted 7 July 2010 \\ Academic Editor: Ashis Basu
}

Copyright ( $) 2010$ Adam D. Brown et al. This is an open access article distributed under the Creative Commons Attribution License, which permits unrestricted use, distribution, and reproduction in any medium, provided the original work is properly cited.

\begin{abstract}
Werner syndrome is a rare disorder that manifests as premature aging and age-related diseases. WRN is the gene mutated in WS, and is one of five human RecQ helicase family members. WS cells exhibit genomic instability and altered proliferation, and in vitro studies suggest that WRN has a role in suppressing homologous recombination. However, more recent studies propose that other RecQ helicases (including WRN) promote early events of homologous recombination. To study the role of WRN helicase on spontaneous homologous recombination, we obtained a mouse with a deleted WRN helicase domain and combined it with the in vivo pink-eyed unstable homologous recombination system. In this paper, we demonstrate that WRN helicase is not necessary for suppressing homologous recombination in vivo contrary to previous reports using a similar mouse model.
\end{abstract}

\section{Introduction}

Werner syndrome (WS) is a rare autosomal recessive disease associated with premature age-related phenotypes such as cancer, osteoporosis, diabetes mellitus and early graying of the hair (review [1]). The gene responsible for WS (WRN) is one of a five human RecQ helicases including BLM, RECQL1, RECQL4, and RECQ5. Like WS, the absence of BLM and RECQL4 gives rise to the clinically distinct diseases, Bloom's syndrome (BS) and Rothmund-Thomson syndrome, respectively. Although a variety of different WRN mutations have been discovered, many result in a truncated nonfunctional WRN (summarized in [2]). Cells from WS patients depict an aging phenotype including reduced proliferation associated with an increase in S-phase [3] and early passage senescence $[3,4]$. Furthermore, WS cells show increased levels of genomic instability thought be caused from increased levels of illegitimate recombination. These observations lead us to investigate the role of WRN in vivo.

For this study we used a WRN mouse model with a deleted helicase domain [5] in combination with the wellestablished murine pink-eyed unstable $\left(p^{\text {un }}\right)$ mouse model that can be used to determine changes in the spontaneous frequency of somatic homologous recombination (HR) events [6-8]. Though rare, this particular Wrn mutation has been found in a small population of WS patients $[9,10]$ and is therefore relevant to the human disease. The $p^{\text {un }}$ assay is based on an HR-mediated deletion of one copy of a $70 \mathrm{~kb}$ DNA duplication that encompasses exons 6-18 of the $p$ gene [11]. The exact deletion of one copy of the repeated region will restore the function of this pigmentation gene, and this can be observed as somatic events in pigmented tissues such as the fur and the retinal pigment epithelium (RPE) $[8,12]$. The further development of the $p^{\text {un }}$ eye spot assay which identifies $p^{\text {un }}$ reversion events on a monolayer of clear RPE cells has proven to be significantly more sensitive and informative than the fur spot assay [6].

Studies in yeast using a similar duplication/deletion assay to the $p^{\text {un }}$ reversion assay have identified several possible mechanisms of HR that may mediate this type of deletion event. These include intrachromatid exchange, one-sided strand invasion, unequal sister chromatid exchange (SCE), sister chromatid conversion, and single-strand annealing (SSA) [13]. Excluding SSA, each of these HR mechanisms 


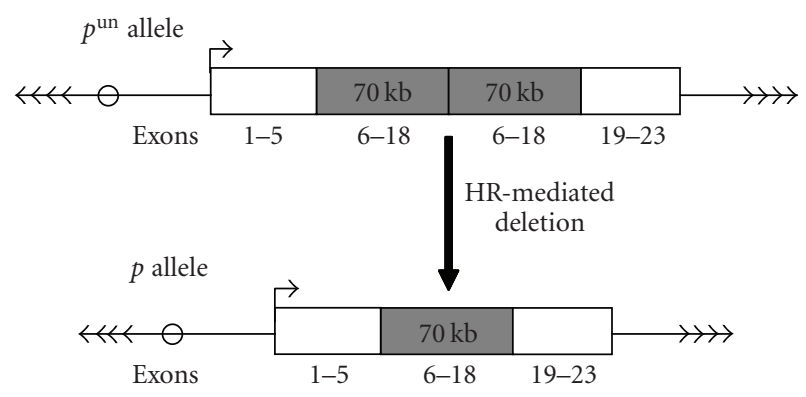

FIGURE 1: HR-mediated reversion of the $p^{\text {un }}$ allele. Schematic of the $p^{\text {un }}$ mutation (tandem duplication of exons 6-18), where an HR event mediates the deletion of one copy rendering a functional $p$ gene allowing pigmentation of RPE cells. Circles and arrowheads represent centromere and telomere DNA, respectively.

is considered RAD51-dependent. RAD51-dependent HR is considered a high fidelity DNA repair mechanism that is frequently associated with DNA replication (review [14]). For example, replication forks can stall or collapse when the replication machinery encounters DNA damage like a single strand break, and HR is capable of repairing the damaged DNA template and restoring the replication fork [15]. Claybon et al. recently reported that in the absence of PARP1, somatic HR events, measured by the $p^{\text {un }}$ system, are highly elevated and that a majority of these events were large clonally expanded cells [16]. These authors went on to suggest that these clonally expanded populations of cells are the result of an HR-mediated deletion that occurred during replication, probably in a RAD51-dependent mechanism (as compared to SSA). The utility of the helicase domain of mouse WRN is discussed below.

\section{Materials and Methods}

2.1. Mouse Lines and PCR Genotyping. WRN helicase mutant $\left(W r n^{\Delta \mathrm{hel} /+}\right)[5]$ mice on an FVB strain background were obtained from Dr. P. Leder, and C57BL/6J and C57BL/6J $p^{\text {un }} /$ un mice were obtained from the Jackson Laboratory (Bar Harbor, ME). In order to obtain congenic C57BL/6J $p^{\text {un } / \text { un }} W r n^{\Delta \text { hel } /+}$ mice (hereafter called $W r n^{\Delta \mathrm{hel} /+}$ ), Wrn $n^{\Delta \mathrm{hel} /+}$ mice were backcrossed 5 times to $\mathrm{C} 57 \mathrm{BL} / 6 \mathrm{~J}$ followed by two additional crosses to $\mathrm{C} 57 \mathrm{BL} / 6 \mathrm{~J} p^{\text {un/un }}$ mice. All mice were maintained with $p^{\text {un/un }}$ mutation. Control $\left(\mathrm{Wrn}^{+/+}\right)$ and experimental $\left(W r n^{\Delta \mathrm{hel} / \Delta \mathrm{hel}}\right)$ animals result from crossing $W r n^{\Delta \mathrm{hel} /+}$ mice together. The $p^{\text {un/un }}$ allele was genotyped by the identification of the phenotypic dilute coat color. Genotypes for the Wrn allele were determined by a PCR amplification protocol obtained from Aya Leder, Harvard Medical School, MA consisting of the following 3 primers: (1) 5'-GTTTCCTCTATCATCTGTAACAGG3', (2) 5'-GCGAAGGAGCAAAGCTGCTAT-3' and (3) 5' AGTGAGACATGTATGACTACC- $3^{\prime}$ and the thermo profile: 1 cycle of $94^{\circ} \mathrm{C}$ for $5 \mathrm{~min} ; 30$ cycles of $94^{\circ} \mathrm{C}$ for $30 \mathrm{~s}, 60^{\circ} \mathrm{C}$ for $30 \mathrm{~s}$, and $72^{\circ} \mathrm{C}$ for $1 \mathrm{~min} ; 1$ cycle of $72^{\circ} \mathrm{C}$ for $3 \mathrm{~min}$. Amplicon size for the wt PCR product is $350 \mathrm{bp}$ and $\mathrm{Wrn}$ mutant $450 \mathrm{bp}$. When necessary, genomic DNA was isolated from fixed RPE using the Qiagen DNeasy Blood and Tissue kit according to manufacture's recommendations.

2.2. Dissection, Visualizing, and Scoring Eye Spots on the Retinal Pigment Epithelium. Harvesting of the eye and dissection of the RPE were carried out as previously described in [7]. RPE whole mounts were visualized and imaged using a Zeiss Lumar V.12 stereomicroscope, Zeiss Axiovision MRm camera, and Zeiss Axiovision 4.6 software (Thornwood, $\mathrm{NY}) \cdot p^{\text {un }}$ reversion events were identified on the transparent monolayer of the RPE as pigmented cells or eye spots. Total number of eye spots and number of cells making up that eye spot were recorded for each RPE according to the criteria set forth by Bishop et al. in [7]. Additionally, the relative distance from the optic nerve of each eye spot was recorded. This was done by using the measurement tool in Adobe Photoshop, by first measuring from the center of the optic nerve to the proximal edge of the eye spot and then from the center of the optic nerve to the edge of the RPE. The relative distance is then determined by dividing the former by the latter.

2.3. Statistical Analysis. All statistics were carried using GraphPad Prism (La Jolla, CA). These include tests for normality (Shapiro-Wilk test), equal variances (Fmax test), two group comparisons (Mann-Whitney test), and contingency tables (Fisher's exact test).

\section{Results}

3.1. Loss of WRN Helicase Activity Does Not Affect the Overall Frequency of Spontaneous Homologous Recombination in Mouse RPE In Vivo. The frequency of spontaneous HR for mice with helicase domain-deficient WRN protein was previously reported as being increased 2 -fold using the $p^{\text {un }}$ fur spot assay [17]. Though the $p^{\text {un }}$ fur spot assay can be considered a faithful assay for measuring HR frequency in vivo, the $p^{\text {un }}$ eye spot assay affords many advantages, including being more sensitive to changes in HR frequency [8] and can reveal information about the timing of events during development [7], developmental patterning [18], and even information about whether the HR events are associated with replication [16]. Therefore, we set out to recapitulate the fur spot study and to determine whether we might be able to reveal any additional phenotypes associated with the $\mathrm{WRN}^{\Delta \text { hel }} \mathrm{HR}$ events. Surprisingly, when we compared the number of eye spots per RPE in $\mathrm{Wrn}^{+/+}$versus $\mathrm{Wr}^{\Delta \mathrm{hel} / \Delta \mathrm{hel}}$ (Table 1 and Figure 2(a)), we were unable to detect a significant increase in the overall frequency of HR events $(P=$ .35, Mann-Whitney test) (Figure 2(b)). The nonparametric Mann Whitney test was used because our data was found to be not normal (data not shown) with unequal variances using a Fmax test $(P<.0001)$. Of interest, the variance within the $\mathrm{Wrn}^{+/+} \mathrm{RPE}$ was larger than expected due to three RPEs with higher than usual numbers of reversion events. To determine whether the lack of difference in HR frequency between $\mathrm{Wrn}^{+/+}$and $W r n^{\Delta \mathrm{hel} / \Delta \text { hel }}$ was due to the wild-type RPE with elevated HR frequency, we compared the frequency of eye spots of our WRN wild-type RPEs with an 
TABLE 1: Summary of RPE examined and $p^{u n}$ reversion frequency by Wrn genotype.

\begin{tabular}{lllll}
\hline Genotype & $\begin{array}{l}\text { Number of } \\
\text { RPE }\end{array}$ & $\begin{array}{l}\text { Total } \\
\text { number of } \\
\text { eye spots }\end{array}$ & $\begin{array}{l}\text { Avg. } \\
\text { number of } \\
\text { eye spots } \\
\text { per RPE }\end{array}$ & $\begin{array}{l}\text { Avg. eye } \\
\text { spot size } \\
\text { (cell } \\
\text { number) }\end{array}$ \\
\hline$W r n^{+/+}$ & 53 & 522 & 10 & 6 \\
$W r n^{\Delta \text { hel } / \Delta \text { hel }}$ & 20 & 152 & 8 & 4 \\
\hline
\end{tabular}

independent wild-type data set that was recently reported by our laboratory [16]. No statistical difference in $p^{u n}$ reversion frequency was observed between these two groups of wildtype RPE (data not shown). We therefore combined these wild-type datasets, compared their combined $p^{u n}$ reversion eyespot frequency with $W_{r n}{ }^{\Delta \mathrm{hel} / \Delta \mathrm{hel}}$, and still did not observe any statistical difference between genotypes (Figure 2(b)). Therefore, it appears that WRN helicase activity is not required for $\mathrm{HR}$, and no additional $\mathrm{HR}$ events are instigated by the WRN mutation.

3.2. Single- and Multicell Eye Spots in Mouse RPE Are Not Affected by WRN Helicase. We classify eye spots as having either single (1 cell) or multi-cell ( $\geq 2$ cells) events [8]. Due to the edge-biased proliferation of the RPE [19] and the apparent "position shift" between single-cell and multicell eye spots [7], we speculate that multi-cell (clonally expanded) $p^{\text {un }}$ reversion events are associated with DNA replication (discussed below). Approximately $60 \%$ of eye spots are normally single cell events. Even though we did not observe an overall difference in HR frequency, we wanted to see if WRN helicase activity affected the clonal expansion of $p^{\text {un }}$ reversion events. Here we found no significant difference between single versus multi-cell eye spots when comparing $W_{r n}{ }^{+/+}$versus $W r n^{\Delta \text { hel/ } / \text { hel }} \operatorname{RPE}(P=.39$, Fisher's exact test Figure (3)). These data indicate that the helicase activity of WRN does not affect clonal expansion of mouse RPE cells following HR.

3.3. Distribution of HR Events during RPE Development Is Not Affected by WRN Helicase Mutation. The mouse RPE develops radially outward from the optic nerve with an edgebiased pattern of proliferation [19]. The RPE begins to form in the developing eye cup at $\sim 8.5 \mathrm{dpc}$ and continues through the first week of postnatal development [20,21]. Much like the age of a tree that can be determined using its concentric rings, the retrospective mapping of an eye spot onto an RPE suggests when during development a $p^{\text {un }}$ reversion event occurred [7]. Previously we have reported mutant genotypes that affected either the timing of $p^{\text {un }}$ reversion events during RPE development or the pattern of RPE development by examining eye spot patterns $[6,18]$. In order to determine if WRN helicase function has a role in HR at a specific point during murine development, each RPE was divided into 10 concentric rings where the inner most ring contains the optic nerve depicting the beginning of RPE development $(0.0-0.1)$ to the outer most ring at the edge of the RPE (0.9-1.0).

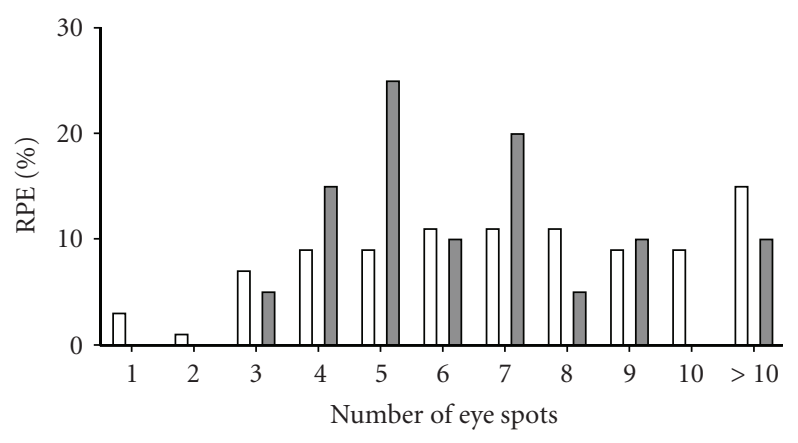

$\square W_{r n}^{+/+}$
$\square W r n^{\Delta \mathrm{hel} / \Delta \mathrm{hel}}$

(a)

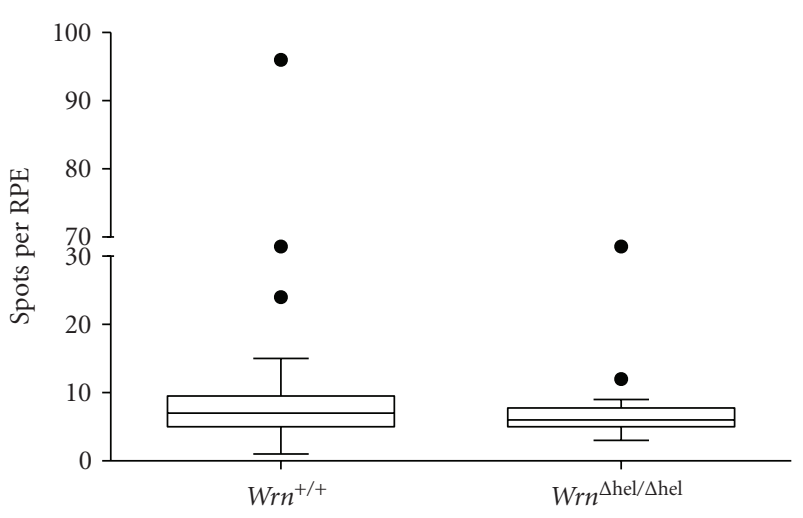

(b)

Figure 2: Frequency of HR in mouse RPE. (a) Population distribution of eye spots per RPE in wild-type (open boxes) and $W r n^{\Delta \text { hel/ } / \text { hel }}$ (grey boxes). (b) Overall frequency of eye spots per RPE shown as a box and whisker plot. No statistical difference in HR was detected between wild-type and $W r n^{\Delta \text { hel/ } / \text { hel }}$ groups $(P=.35)$.

At each interval, the pattern of positional distribution was similar for both $\mathrm{Wrn}^{+/+}$and $\mathrm{Wrn}^{\Delta \mathrm{hel} / \Delta \text { hel }}$ for all eye spots $(P=.22$, Chi-square test, Figure 4$)$. Of note, the positional analysis of the eye spots measures the distance from the center of the optic nerve to the most proximal cell of an eye spot, irrespective of the number of cells that constitutes the eye spot. These results suggest that the effect of the WRN helicase mutation on HR does not alter the timing or distribution of $p^{\text {un }}$ reversion events during mouse RPE.

\section{Discussion}

In summary, mice expressing a helicase-deficient $\mathrm{Wrn}$ allele did not have an increase in the frequency of spontaneous HR. Our results differ substantially from earlier work done using this same mouse model with the less sensitive $p^{\text {un }}$ fur spot assay which observed at least a 2 -fold increase in $p^{\text {un }}$ reversion events [17]. In our experience we have never observed a discrepancy in $p^{\text {un }}$ reversion frequency between the neural crest-derived melanocyte-dependent fur spot assay and the neural epithelium-derived RPE-based eye spot assay. 


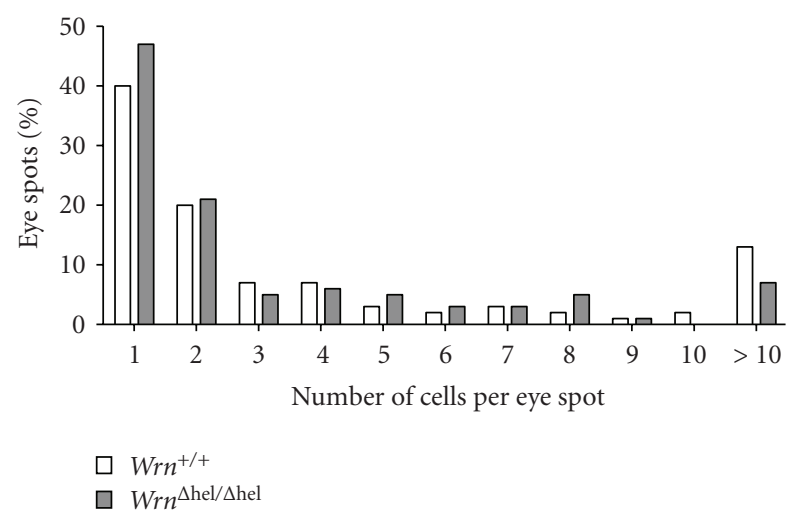

FIGURE 3: Frequency of the different sizes of eye spot (number of cells per eye spot) in mouse RPE. Wrn ${ }^{\Delta \mathrm{hel} / \Delta \mathrm{hel}}$ (grey boxes) does not affect cell size distribution compared to wild-type (open boxes) $(P=.39)$.

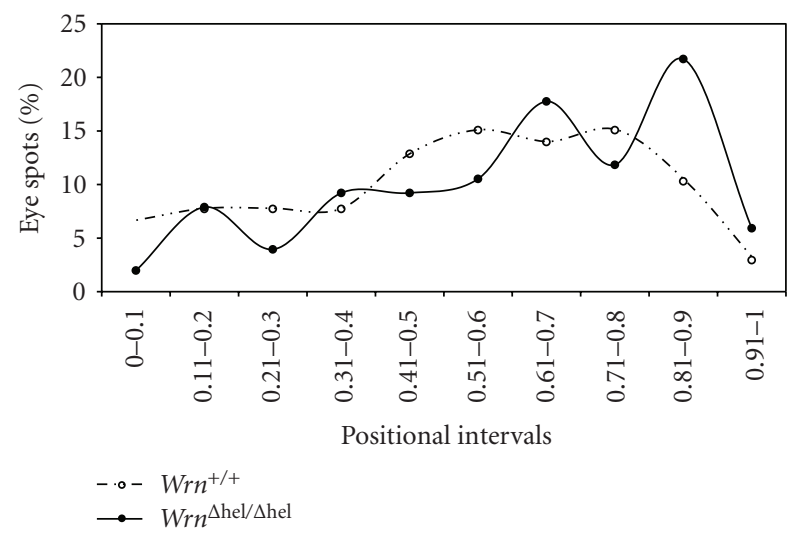

FIgURe 4: Positional distribution of all eye spots. WRN helicase does not affect positional or pattern distribution $(P=.22)$. Wild type (open circles with dash line) and $W r n^{\text {shel/shel }}$ (closed circles with solid line) RPE.

However, it should be noted that we have not examined the frequency of $p^{\text {un }}$ fur spots in our C57BL6/J congenic animals, and it is formally possible that the difference may be due to difference in strain background. Our result also differs from a more recent report using the $p^{\text {un }}$ eye spot assay with the expression of a transgenic dominant negative human WRN allele [22]. This latter report observed a 30\% increase in eye spots (from an average of 6 to 8 eye spots per RPE in wild-type and WRN impaired, resp.), a relatively slight, though significant increase for the $p^{\text {un }}$ eye spot assay, that could be simply explained by sample size; Yamamoto et al. [22] used approximately 50 RPE per group compared to our 20 RPE. Alternately, the difference could be due to a difference in using a mouse helicase deficient Wrn mutant mouse and a mouse model that expressed a transgenic dominant negative allele of human WRN.

The $p^{\text {un }}$ fur spot assay is reliant upon detecting large clonal expansions of melanocytes following $p^{\text {un }}$ reversion that are visible in the fur (at least $1 \mathrm{~mm}^{2}$ ). In contrast, the $p^{\text {un }}$ eye spot assay can detect both single cell and clonally expanded (multi-cell) reversion events. Additionally, the $p^{\text {un }}$ eye spot assay can be used to determine any differences in the timing of HR events during embryonic development. In this study we found that the helicase activity of mouse WRN does not affect the frequency of single versus multi-cell events and nor does it affect the timing of spontaneous HR events during mouse embryonic development.

Although WS cells are described as having genomic instability, there is some discrepancy as to the role WRN plays in HR. In support of WRN as a suppressor of illegitimate HR, WS patients exhibit variegated chromosomal translocations [23], elevated recombination levels between microhomology plasmids [24], approximate 2-fold increase of RAD51 focus formation [25], and sensitivity to agents that lead to replication stress [5, 26-28]. Additionally, WRN is known to associate with proteins tied to replication machinery like RPA [29-32], PCNA [33-35], Pol $\delta[36,37]$, and RAD52 [38]. In contrast though, WS cells do not have elevated amounts of SCE which are the hallmark of BS [39].

More recent studies have begun to show that RecQ helicases (e.g., WRN) promote $\mathrm{HR}$ via mechanisms like DNA resection. Following double-strand breaks (DSBs), the helicase domain of Sgs1 (yeast RecQ orthologue of WRN) is required for resection of DNA ends to produce single strand DNA substrates for RAD51 [40, 41]. Additionally, the helicase function of Sgs1 is required for normal kinetics of $\mathrm{HR}$ at the MAT locus, and yeast mutants for Sgs 1 and Exo1 nuclease exhibit sensitivity to DNA damaging at similar levels to Rad52 mutants (which are diminished for HR repair) [42]. These same authors subsequently went on to demonstrate that the RecQ helicase BLM also has some DNA resection functions following Camptothecin-induced DSBs and that BLM function of DSB resection is in parallel with an EXO1-dependent pathway [42]. A separate study investigated the role of WRN following exposure to chromium(VI), an agent known to induce DSBs, and found that chromium(VI) treated human cells depleted of WRN and WS cells had delayed or absent RAD51 focus formation [43]. This study again suggests that WRN is important for promoting HR, potentially in early steps of this process (e.g., initiation via resection) following DNA damage. Unlike the other human RecQ helicase members, WRN also has exonuclease activity, so understanding which enzymatic function of WRN is involved in promoting $\mathrm{HR}$ is valuable to our knowledge of this protein, as well as insightful to the syndrome. As it pertains to this study, we found that the helicase function of WRN is neither necessary for, nor suppresses spontaneous HR. With regard to other RecQ helicases, we recently found HR to be significantly elevated in the absence of BLM using the $p^{\text {un }}$ eye spot assay (data not shown). Considering the lack of effect observed in our study, and only a mild suppressive effect in the Yamamoto et al. study [21] compared to a deficiency in BLM (data not shown), it would appear that WRN only plays a minor role in suppressing $\mathrm{HR}$, possibly the result of redundancy amongst the different RecQ family members. Together, these studies give insight into the potential differences between two of the known five human RecQ helicases and suggest that future studies are warranted to better understanding the functions of WRN (and BLM) in HR. 


\section{Acknowledgment}

This paper was supported by the National Institutes of Health [K22ES012264 to A. J. R. Bishop] and National Institute of Aging [T32AG021890 A. D. Brown]. A. D. Brown and A. B. Claybon contributed equally to this work.

\section{References}

[1] B. A. Kudlow, B. K. Kennedy, and R. J. Monnat Jr., "Werner and Hutchinson-Gilford progeria syndromes: mechanistic basis of human progeroid diseases," Nature Reviews Molecular Cell Biology, vol. 8, no. 5, pp. 394-404, 2007.

[2] M. Muftuoglu, J. Oshima, C. von Kobbe, W.-H. Cheng, D. F. Leistritz, and V. A. Bohr, "The clinical characteristics of Werner syndrome: molecular and biochemical diagnosis," Human Genetics, vol. 124, no. 4, pp. 369-377, 2008.

[3] F. Takeuchi, F. Hanaoka, M. Goto, M. Yamada, and T. Miyamoto, "Prolongation of S phase and whole cell cycle in Werner's syndrome fibroblasts," Experimental Gerontology, vol. 17, no. 6, pp. 473-480, 1982.

[4] R. G. A. Faragher, I. R. Kill, J. A. A. Hunter, F. M. Pope, C. Tannock, and S. Shall, "The gene responsible for Werner syndrome may be a cell division "counting" gene," Proceedings of the National Academy of Sciences of the United States of America, vol. 90, no. 24, pp. 12030-12034, 1993.

[5] M. Lebel and P. Leder, "A deletion within the murine Werner syndrome helicase induces sensitivity to inhibitors of topoisomerase and loss of cellular proliferative capacity," Proceedings of the National Academy of Sciences of the United States of America, vol. 95, no. 22, pp. 13097-13102, 1998.

[6] A. J. R. Bishop, M. C. Hollander, B. Kosaras, R. L. Sidman, A. J. Fornace Jr., and R. H. Schiestl, "Atm-, p53-, and Gadd45adeficient mice show an increased frequency of homologous recombination at different stages during development," Cancer Research, vol. 63, no. 17, pp. 5335-5343, 2003.

[7] A. J. R. Bishop, B. Kosaras, N. Carls, R. L. Sidman, and R. H. Schiestl, "Susceptibility of proliferating cells to benzo[a]pyrene-induced homologous recombination in mice," Carcinogenesis, vol. 22, no. 4, pp. 641-649, 2001.

[8] A. J. R. Bishop, B. Kosaras, R. L. Sidman, and R. H. Schiestl, "Benzo(a)pyrene and X-rays induce reversions of the pinkeyed unstable mutation in the retinal pigment epithelium of mice," Mutation Research, vol. 457, no. 1-2, pp. 31-40, 2000.

[9] C.-E. Yu, J. Oshima, Y.-H. Fu et al., "Positional cloning of the Werner's syndrome gene,” Science, vol. 272, no. 5259, pp. 258262, 1996.

[10] C.-E. Yu, J. Oshima, E. M. Wijsman et al., "Mutations in the consensus helicase domains of the Werner syndrome gene. Werner's Syndrome Collaborative Group," American Journal of Human Genetics, vol. 60, no. 2, pp. 330-341, 1997.

[11] Y. Gondo, J. M. Gardner, Y. Nakatsu et al., "High-frequency genetic reversion mediated by a DNA duplication: the mouse pink-eyed unstable mutation," Proceedings of the National Academy of Sciences of the United States of America, vol. 90, no. 1, pp. 297-301, 1993.

[12] R. H. Schiestl, F. Khogali, and N. Carls, "Reversion of the mouse pink-eyed unstable mutation induced by low doses of X-rays," Science, vol. 266, no. 5190, pp. 1573-1576, 1994.

[13] A. Galli and R. H. Schiestl, "On the mechanism of UV and $\gamma$-ray induced intrachromosomal recomhination in yeast cells synchronized in different stages of the cell cycle," Molecular and General Genetics, vol. 248, no. 3, pp. 301-310, 1995.
[14] F. Pâques and J. E. Haber, "Multiple pathways of recombination induced by double-strand breaks in Saccharomyces cerevisiae," Microbiology and Molecular Biology Reviews, vol. 63, no. 2, pp. 349-404, 1999.

[15] C. Arnaudeau, C. Lundin, and T. Helleday, "DNA doublestrand breaks associated with replication forks are predominantly repaired by homologous recombination involving an exchange mechanism in mammalian cells," Journal of Molecular Biology, vol. 307, no. 5, pp. 1235-1245, 2001.

[16] A. B. Claybon, B. Karia, C. Bruce, and A. J. R. Bishop, "PARP1 suppresses homologous recombination events in mice in vivo," Nucleic Acids Research, pp. 1-8, 2010.

[17] M. Lebel, "Increased frequency of DNA deletions in pink-eyed unstable mice carrying a mutation in the Werner syndrome gene homologue," Carcinogenesis, vol. 23, no. 1, pp. 213-216, 2002.

[18] A. J. R. Bishop, B. Kosaras, M. C. Hollander, A. Fornace Jr., R. L. Sidman, and R. H. Schiestl, "p21 controls patterning but not homologous recombination in RPE development," DNA Repair, vol. 5, no. 1, pp. 111-120, 2006.

[19] L. Bodenstein and R. L. Sidman, "Growth and development of the mouse retinal pigment epithelium. I. Cell and tissue morphometrics and topography of mitotic activity," Developmental Biology, vol. 121, no. 1, pp. 192-204, 1987.

[20] A. V. Ershov and O. G. Stroeva, "Post-natal pattern of cell proliferation in retinal pigment epithelium of mice studied with tritiated thymidine autoradiography," Cell Differentiation and Development, vol. 28, no. 3, pp. 173-177, 1989.

[21] A. Nakayama, M.-T. T. Nguyen, C. C. Chen, K. Opdecamp, C. A. Hodgkinson, and H. Arnheiter, "Mutations in microphthalmia, the mouse homolog of the human deafness gene MITF, affect neuroepithelial and neural crest-derived melanocytes differently," Mechanisms of Development, vol. 70, no. 1-2, pp. 155-166, 1998.

[22] M. L. Yamamoto, R. Reliene, J. Oshima, and R. H. Schiestl, "Effects of human Werner helicase on intrachromosomal homologous recombination mediated DNA deletions in mice," Mutation Research, vol. 644, no. 1-2, pp. 11-16, 2008.

[23] D. Salk, K. Au, H. Hoehn, and G. M. Martin, "Cytogenetics of Werner's syndrome cultured skin fibroblasts: variegated translocation mosaicism," Cytogenetics and Cell Genetics, vol. 30, no. 2, pp. 92-107, 1981.

[24] R. Z. Cheng, S. Murano, B. Kurz, and R. J. Shmookler Reis, "Homologous recombination is elevated in some Wernerlike syndromes but not during normal in vitro or in vivo senescence of mammalian cells," Mutation Research, vol. 237, no. 5-6, pp. 259-269, 1990.

[25] P. Pichierri, A. Franchitto, P. Mosesso, and F. Palitti, "Werner's syndrome protein is required for correct recovery after replication arrest and DNA damage induced in S-phase of cell cycle," Molecular Biology of the Cell, vol. 12, no. 8, pp. 24122421, 2001.

[26] R. Elli, L. Chessa, A. Antonelli, P. Petrinelli, R. Ambra, and L. Marcucci, "Effects of topoisomerase II inhibition in lymphoblasts from patients with progeroid and "chromosome instability" syndromes," Cancer Genetics and Cytogenetics, vol. 87, no. 2, pp. 112-116, 1996.

[27] M. Okada, M. Goto, Y. Furuichi, and M. Sugimoto, "Differential effects of cytotoxic drugs on mortal and immortalized Blymphoblastoid cell lines from normal and Werner's syndrome patients," Biological \& Pharmaceutical Bulletin, vol. 21, pp. 235-239, 1998. 
[28] M. Poot, K. A. Gollahon, and P. S. Rabinovitch, "Werner syndrome lymphoblastoid cells are sensitive to camptothecininduced apoptosis in S-phase," Human Genetics, vol. 104, no. 1, pp. 10-14, 1999.

[29] J.-C. Shen, M. D. Gray, J. Oshima, and L. A. Loeb, "Characterization of Werner syndrome protein DNA helicase activity: directionality, substrate dependence and stimulation by replication protein A," Nucleic Acids Research, vol. 26, no. 12, pp. 2879-2885, 1998.

[30] R. M. Brosh Jr., D. K. Orren, J. O. Nehlin et al., "Functional and physical interaction between WRN helicase and human replication protein A," Journal of Biological Chemistry, vol. 274, no. 26, pp. 18341-18350, 1999.

[31] K. M. Doherty, J. A. Sommers, M. D. Gray et al., "Physical and functional mapping of the replication protein A interaction domain of the Werner and Bloom syndrome helicases," Journal of Biological Chemistry, vol. 280, no. 33, pp. 29494-29505, 2005.

[32] J.-C. Shen, Y. Lao, A. Kamath-Loeb, M. S. Wold, and L. A. Loeb, "The N-terminal domain of the large subunit of human replication protein A binds to Werner syndrome protein and stimulates helicase activity," Mechanisms of Ageing and Development, vol. 124, no. 8-9, pp. 921-930, 2003.

[33] M. Lebel, E. A. Spillare, C. C. Harris, and P. Leder, "The Werner syndrome gene product co-purifies with the DNA replication complex and interacts with PCNA and topoisomerase I," Journal of Biological Chemistry, vol. 274, no. 53, pp. 37795-37799, 1999.

[34] S. Huang, S. Beresten, B. Li, J. Oshima, N. A. Ellis, and J. Campisi, "Characterization of the human and mouse WRN $3^{\prime} \rightarrow 5^{\prime}$ exonuclease," Nucleic Acids Research, vol. 28, no. 12, pp. 2396-2405, 2000.

[35] A. M. Rodríguez-López, D. A. Jackson, J. O. Nehlin, F. Iborra, A. V. Warren, and L. S. Cox, "Characterisation of the interaction between WRN, the helicase/exonuclease defective in progeroid Werner's syndrome, and an essential replication factor, PCNA," Mechanisms of Ageing and Development, vol. 124, no. 2, pp. 167-174, 2003.

[36] A. S. Kamath-Loeb, E. Johansson, P. M. J. Burgers, and L. A. Loeb, "Functional interaction between the Werner syndrome protein and DNA polymerase $\delta$," Proceedings of the National Academy of Sciences of the United States of America, vol. 97, no. 9, pp. 4603-4608, 2000.

[37] A. S. Kamath-Loeb, L. A. Loeb, E. Johansson, P. M. J. Burgers, and M. Fry, "Interactions between the Werner syndrome helicase and DNA polymerase $\delta$ specifically facilitate copying of tetraplex and hairpin structures of the d(CGG)n trinucleotide repeat sequence," Journal of Biological Chemistry, vol. 276, no. 19, pp. 16439-16446, 2001.

[38] K. Baynton, M. Otterlei, M. Bjørås, C. Von Kobbe, V. A. Bohr, and E. Seeberg, "WRN interacts physically and functionally with the recombination mediator protein RAD52," Journal of Biological Chemistry, vol. 278, no. 38, pp. 36476-36486, 2003.

[39] M. I. Melaragno, D. Pagni, and M. D. A. C. Smith, "Cytogenetic aspects of Werner's syndrome lymphocyte cultures," Mechanisms of Ageing and Development, vol. 78, no. 2, pp. 117122, 1995.

[40] E. P. Mimitou and L. S. Symington, "Sae2, Exo1 and Sgs1 collaborate in DNA double-strand break processing," Nature, vol. 455, no. 7214, pp. 770-774, 2008.

[41] Z. Zhu, W.-H. Chung, E. Y. Shim, S. E. Lee, and G. Ira, "Sgs1 helicase and two nucleases Dna2 and Exo1 resect DNA doublestrand break ends," Cell, vol. 134, no. 6, pp. 981-994, 2008.
[42] S. Gravel, J. R. Chapman, C. Magill, and S. P. Jackson, "DNA helicases Sgs1 and BLM promote DNA double-strand break resection," Genes and Development, vol. 22, no. 20, pp. 27672772, 2008.

[43] A. Zecevic, H. Menard, V. Gurel, E. Hagan, R. DeCaro, and A. Zhitkovich, "WRN helicase promotes repair of DNA double-strand breaks caused by aberrant mismatch repair of chromium-DNA adducts," Cell Cycle, vol. 8, no. 17, pp. 27692778, 2009. 

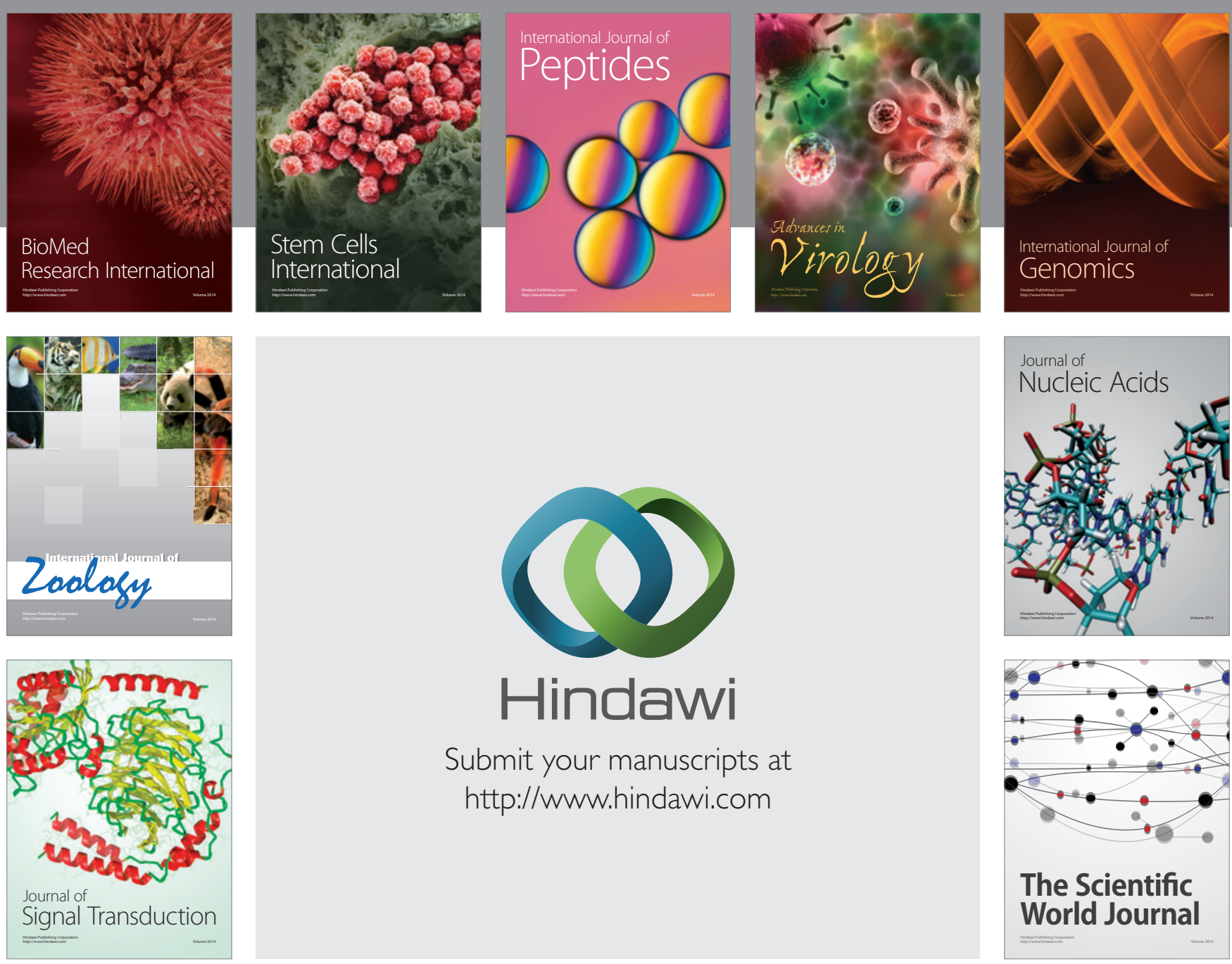

Submit your manuscripts at

http://www.hindawi.com
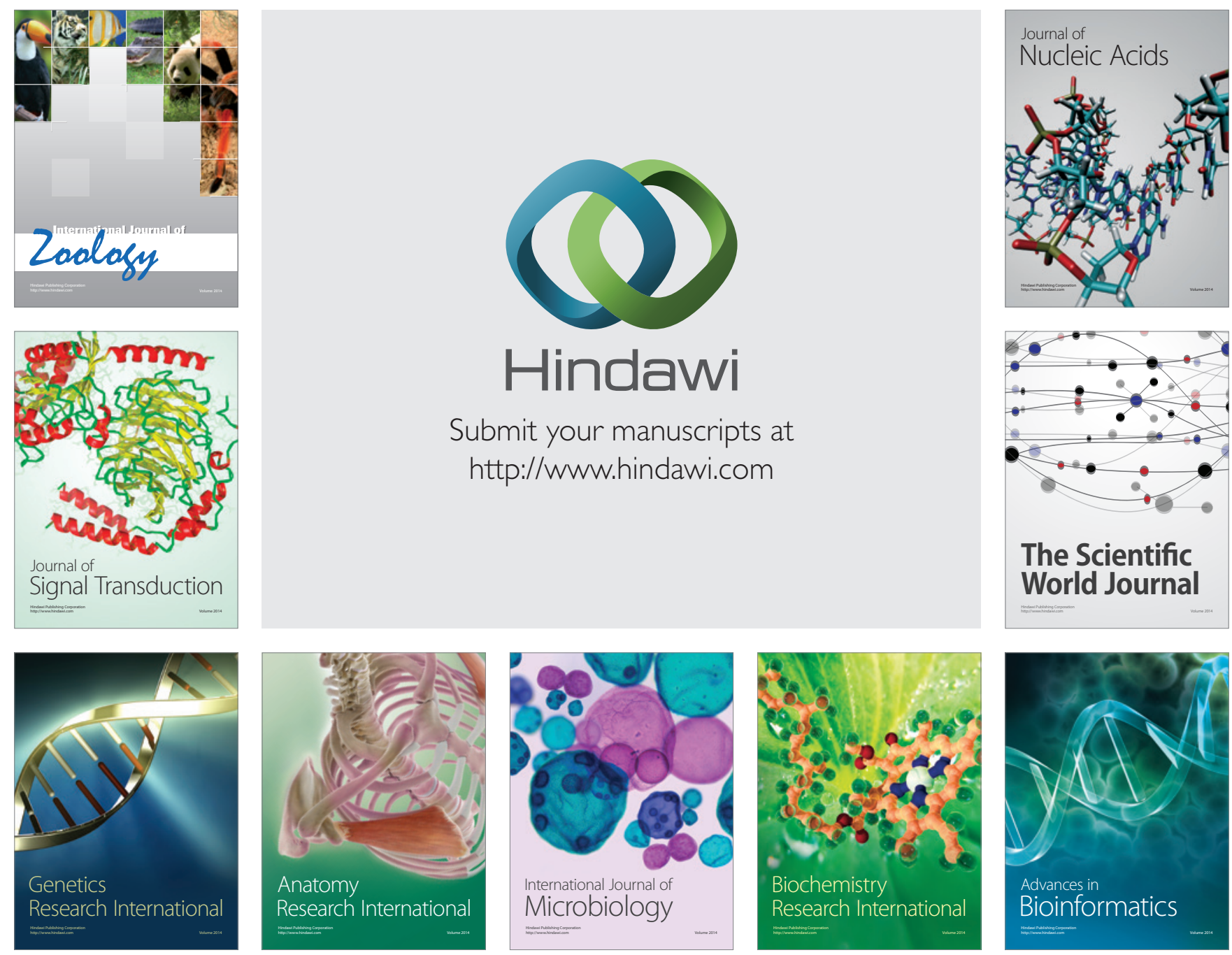

The Scientific World Journal
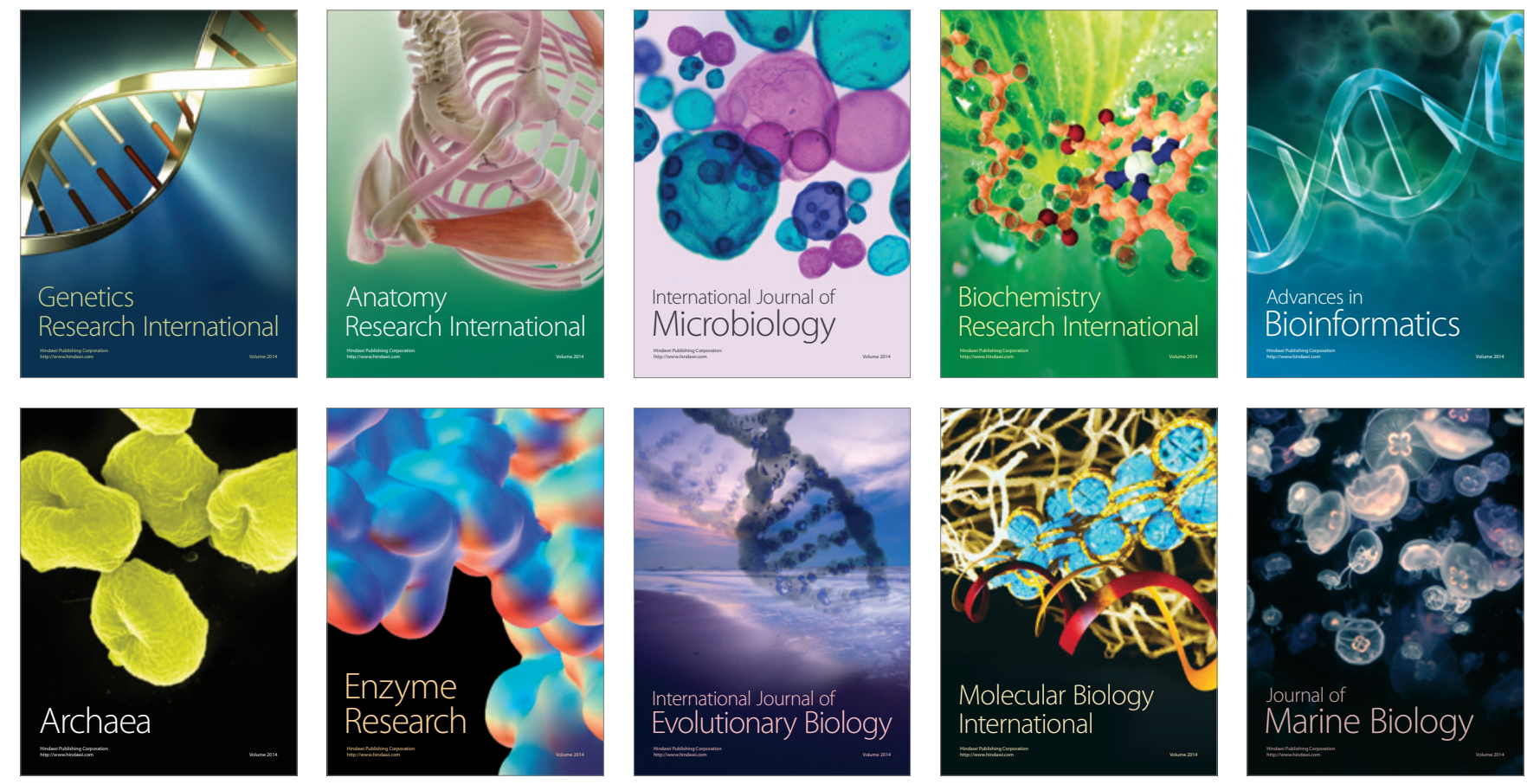